\title{
REVIEW OF DYNAMIC INSTABILITIES IN CIRCULAR ACCELERATORS*
}

\author{
V. Kelvin Neil
}

Lawrence Radiation Laboratory, University of California Livermore, California

\section{Summary}

The coherent electromagnetic self-forces of a beam of particles in an accelerator can render unstable the motion of individual particles, or give rise to unstable coherent motion of the beam. The latter is the subject of this work. Coherent longitudinal bunching of an azimuthally uniform beam can result from the negative mass instability or interaction of the beam with the accelerating cavities. 'The negative mass instability can be suppressed by a sufficient spread in circulation frequency or by surrounding the beam with appropriate walls. The beam-cavity interaction can be overcome by careful design of the rf system. The interaction of the self-fields with the surrounding walls can lead to unstable coherent transverse motion through the finite resistance of the wall material. This instability may be suppressed by a sufficient spread in betatron frequencies. The relatively slow growth rate also allows stabilization by means of electronic feedback, and the stabilizing effect of passive structures has also been investigated. Experimental and theoretical investigation has led to an understanding of these effects to the extent that accelerators can be designed to overcome the instabilities.

\section{Introduction}

All beam-induced instabilities in particle accelerators have a common ultimate result they limit the number of particles that can be accelerated in the machine. It is unwise to design a new accelerator and attempt to predict an achievable current without considering all known limitations. The purpose of this work is to make the reader more familiar with some of these limiting effects and the degree of understanding that has been obtained with regard to them. In plasma physics new experimental devices are designed to circumvent instabilities as they are discovered and investigated. It is also possible to apply this philosophy to the design of particle accelerators.

In this work I shall catalogue several instabilities that arise from the coherent electromagnetic self-forces of the beam. These forces can render unstable the motion of individual particles, or result in unstable coherent motion of the entire beam. The former has been rather thoroughly investigated, and will not be discussed. Rather, I

*Work performed under the auspices of the U. S. Atomic Energy Commission. To be submitted to the 1967 U.S. National Particle Accelerator Conference, March 1-3, 1967, Washington, D. C. Invited abstract. shall concentrate on effects that result in unstable motion of the beam as a whole. No attempt will be made to familiarize the rcader with the mathematical details of the treatment of various instabilities. The calculational techniques used are straightforward (at least in principle) and wellknown to plasma physicists. When possible, a simple physical explanation will be given for each instability. I shall discuss the dependence of the growth rates on the parameters of the marhine, and present methods whereby the instability may be suppressed. The presentation draws on the work of many theoretical and experimental physicists throughout the world, and I shall not attempt to give complete references. A comprehensive bibliography has been given by Sessler ${ }^{1}$ in a paper similar to this one, and the references of this paper are limited primarily to work published or performed subsequently.

Taking a positive approach to the problem, I shall devote the next section to a general discussion of methods for suppressing instabilities. These methods will be illustrated in more detail when applied to the specific instabilities in Sections 3 and 4 . Two phenomena that result in unstable longitudinal motion, the negative mass effect and the interaction between the beam and the rf cavity, are discussed in Section 3 . The negative mass instability is a particularly good example because it is easily understood physically and can be suppressed by several methods. Transverse resistive instabilities in both bunched and unbunched beams are discussed in Section 4. Section 5 contains a few observations regarding coherent effects in storage rings and Section 6 contains some general conclusions about the present state of theoretical understanding.

\section{Methods for Suppressing Instabilities}

A beam of particles may undergo unstable motion for a variety of reasons, and some of these will be apparent in the following sections. The unstable motion falls generally into two categories: either the beam undergoes unstable transverse oscillations, or unstable longitudinal motion results in a longitudinal density fluctuation. The diverse methods of stabilizing the motion are discussed below.

\section{Landau Damping}

Historically, the first method investigated for suppressing instabilities in accelerators is the mechanism of Landau damping, which relies on a spread in some parameter within the bcam. If the beam is subject to unstable longitudinal oscillations, a spread in circulation frequency is effective. This spread may arise from a spread in energy or in betatron oscillation amplitudes. ${ }^{2}$ 
If the beam is subject to unstable transverse oscillations, then a sprcad in the betatron frequency is effective. Theoretically, a criterion exists for each instability, and this criterion places a lower limit on the spread necessary to suppress the instability. This lower limit always depends upon the beam current as well as the parameters of the machine. Thus, when Landau damping is relied upon as the sole method for suppressing a particular instability, the spread in the appropriate quantity may be insufficient for stability at some current level. When this level is exceeded the bcam becomes unstable, and the entire beam, or a portion of it, is lost. In some instances it is possible to artificially introduce additional spread, suppress the instability, and achieve a higher beam intensity.

\section{Altering the Bcam's Environment}

Many instabilities may be suppressed by methods that do not rely on a frequency sprcad within the beam. These methods can render the beam stable in the absence of any spread, and their effectiveness is independent of beam intonsity. These methods may alter the configuration of the equilibrium electromagnetic fields (either the external guide field or the coherent self-fields of the beam) in such a way as to render the beam stable under certain perturbations. A simple, though not useful, example of this is the stabilization of longitudinal density fluctuations in an azimuthally uniform beam. Above the transition cnergy, these fluctuations can lead to the negalive mass instability, which will be discussed in detail in the next section. But it is possible, in principle at lcast, to alter the magnetic guide field so that the beam is always below transition and the instability does not occur. In Section 4 more practical examples of this incthod will be given. Dekanskij and Skrinski ${ }^{3}$ as well as Courant and Sessler 4 have shown that a proper choice of axial betatron wave number $\nu$ can stabilize the vertical motion of a single bunch of particles. In addition, the analysis of Ref. 4 shows how the value of $\nu$ can determine the unstable normal modes of a beam with more than one bunch.

Another method of stabilizing coherent motion is to alter the fields arising from the perturbation (not the equilibrium fields) in such a way as to render the motion stable or cause the perturbation to decay. This can often be accomplished by surrounding the beam with an appropriate structure consisting of passive clectronic circuits. This method draws upon the vast technology that has been developed by workers in the field of traveling wave tubes. Although these workers have been concerned with structures that render a bcam unstable and thus form the basis for amplification, similar techniques can be applied in accelerators to insure stability. In the case of the negative mass instability, TWT structures are directly applicable, as will bo shown in the next section. The presence of the wrong kind of external circuits, on the other hand, can have an extremely adverse effect on beam stability. In particular, if clearing electrodes are to be used in a machine, care must bo taken to insure that this circuit is properly
terminated.

\section{Stabilization by Feedback}

A spectacularly successful method for suppressing transverse instabilities has been used in several accolerators. This method is related to the previous one, except that the external circuits are not passive. It is the method of leodback. Transverse motion of the bcam is detected electronically, the signal is amplified, then fed back to the beam in such a phase as to damp the transverse oscillations. As discussed in Section 4, transverse instabilities result from resistive losses in the surroundings of the beam. The growth rate is generally quite long, of the order of milliseconds, and it is just this long growth time that allows the fecdback mechanism time to work. In a broad sense, the feedback circuit can be rogarded as supplying a negative resistance.

\section{Enstable Longitudinal Motion}

In this section two examples of instabilities that result in a longitudinal bunching in the beam are discussed. First we present the negative mass instability, and second the instability that can result lrom the interaction of an azimuthally uniform beam with a rf cavity through which it passes. Either of these cffects can occur in a bunched beam, and in practice do, but the theory is much more simple for an unbunched beam.

\section{Negative Mass Instability}

The negative mass instability has the destinction of being one of the vcry few dynamic effects in accelerators to be predicted theoretically before it was observed. The physical explanation is quite simple. Suppose that a beam, which has uniform density in the azimuthal direction, is subject to a perturbation that locally increases the charge density. Particlos at the front of the region of increased charge feel a force that tends to accclerate them. But if the circulation frequency is a decreasing function of energy, those particles move out radically, lose azimuthal velocity, and fall back toward the center of the bunch. Similarly particles at the rear of the bunch move inward radially, increase their azimuthal velocity and move forward toward the center of the bunch. The azimuthal bunching limits the intensity of the accelerator by reducing the capture efficiency of the rf system. If the bunching persists after capture, the interaction of the bunches causes loss of particles from the stable phase region.

For a density fluctuation with azimuthal $(\theta)$ and time dependence exp $i(n \theta-\omega t)$ in a beam with no frequency spread, the analysis yields the following expression for w:

$$
\frac{\omega}{n}=\omega_{c} \pm\left[\frac{e^{2} N g \omega_{c} \partial \omega_{c}}{2 \pi \gamma^{2} R \partial E}\right]^{1 / 2}
$$


where $\omega_{c}$ is the angular circulation frequency, e is the electron charge, $R$ is the major radius of the beam, $\gamma$ is the particles energy in units of the rest energy, and $\mathrm{E}$ is their energy. The total number of particles in the bearn is $\mathrm{N}$, and $\mathrm{g}$ is a function of $\gamma$ as well as the geometry of the beam and vacuum tank. For perfectly conducting walls, the quantity $\mathrm{g}$ is independent of $\gamma$ and always positive. We see that above the transition energy where $\partial \omega_{\mathrm{c}} / \mathrm{\partial E}$ is negative, one of the roots of Eq. (1) corresponds to an cxponentially growing wave.

If the instability is to be suppressed by Landau damping alone, a spread in circulation frequency $\Delta \omega_{c}$ is required. The lower limit on the spread necessary is given by

$$
\Delta \omega_{\mathrm{c}}=\mathrm{K} \operatorname{Im} \omega / \mathrm{n} \text {, }
$$

where the imaginary part of $\omega$ is found from Eq. (1) with $\partial \omega_{\mathrm{C}} / \partial \mathrm{E}<0$. The quantity $\mathrm{K}$ in $\mathrm{Eq}$. (2) is a constant of order unity. Its exact value depends on the form of the frequency distribution of the particles. Physically the criterion states that in order to suppress the instability, the spread in frequency must be great enough to allow particles to sweep through the bunch in a time comparable to the growth rate in the absence of damping.

It is not always convenient or even possible to provide enough frequency spread to satisfy $\mathrm{Eq}$. (2). But notice that in general, the quantity $g$ depends on the energy of the particles. With the proper choice of walls, g can be made positive or negative. 6,7 In fact, if the walls are properly constructed, the energy dependence of $g$ makes it possible for $g$ to change sign from positive to negative right at the transition energy. Thus the product $g \partial \omega_{\mathrm{c}} / \partial \mathrm{E}$ remains always positive, and the instability is suppressed throughout the acceleration cycle.

The possibility of constructing walls that result in a negative value for $g$ follow s directly from TWT technology. In a TWT the particles have positive longitudinal mass, and structures were sought that would render the se beams unstable to longitudinal bunching. Such devices fall into the general category of inductive wall amplifiers. It need only be noted that a structure that makes a positive mass beam unstable will in turn stabilize a negative mass beam. Several such structures are described in Refs. 6 and 7. Typical of these structures is the helical ribbon surrounding the beam, as shown in Fig. 1. For this structure we have

$$
g=2\left[\frac{\ln (b / a)}{\gamma^{2}}-\frac{\beta^{2}}{2 \alpha^{2}}\right],
$$

in which the unperturbed velocity of the particles is $\beta c$, and the approximation $\sin ^{2} \alpha=\alpha^{2}$ has been used. All other quantities are defined in the figure, We see that $g<0$ if the condition

$$
\gamma^{2}>1+2 \alpha^{2} \ln (\mathrm{b} / \mathrm{a})
$$

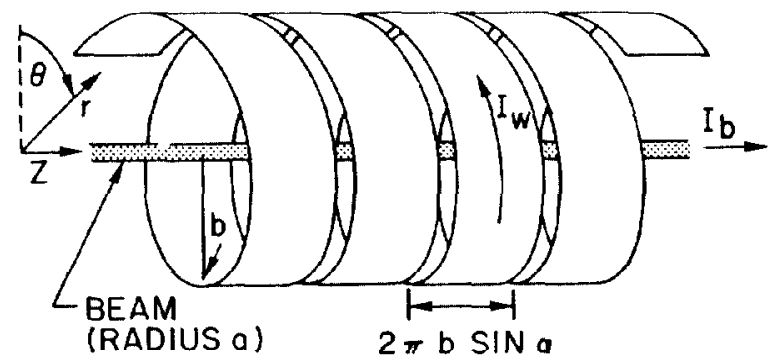

Fig. 1 Helical conductor for suppressing the negative mass instability. The structure forces the image current, $I_{W}$, to flow in a circuitous path, thus enhancing the magnetic field of the beam.

is satisfied. Although this result is based on a rather idealized analysis and the value of the beam radius, a, is not under strict control, it is apparently possible to choose $\alpha$, $a$, and $b$ such that $g$ changes sign from positive to negative when $\gamma=\gamma_{t}$, where $\gamma_{t}$ is the value at the transition energy. For machines in which $\gamma>\gamma_{t}$ at all stages of the cycle, it suffices to choose $a, a$ and $b$ such that $\mathrm{g}$ is always negative. It must be emphasized that the theory of Refs. 6 and 7 is applicable only for perturbation wavelengths $\lambda$ such that $\lambda \gg b$.

In concluding this discussion we note that if the walls have finite conductivity, the quantity $g$ has an imaginary part, and Eq. (1) will always have a root with a positive imaginary part, corresponding to an exponentially growing wave. This so-called longitudinal resistive wall instability has been predicted theoretically, but never really identified experimentally in an accelerator. The instability arises from the same phenomena that is utilized in the resistive wall amplifier.

\section{Beam - rf Cavity Interaction}

The interaction of the bean with an rf cavity or cavities manifests itself in many ways. One of these is simply referred to as beam loading. The term conventionally covers the effect of the presence of the beam on the cavity and its driving mechanism during the normal accelerating cycle. Though not a dynamic instability, an undesirable consequence of the effect can occur during the capture process. In steady state, the back voltage induced by the beam is equal to the product of the shunt impedance of the cavity and the Fourier component of the beam that is in resonance with the cavity. This is not generally true during the transients of the capture process, but the induced voltage can be appreciable. The accelerating rf voltage is usually turned on slowly in order to capture adiabatically the particles already injected into the machine. If the shunt impedance is too large, the induced voltage may become large enough to significantly alter the total voltage, and the entire capture process is disrupted. An obvious way to alleviate the problem is to reduce the shunt impedance, at least during the capture process. 
As an example of a dynamic instability arising from the beam-cavity interaction, consider an azimuthally uniform beam and a rf cavity through which it passes. For purposes of this discussion, the cavity is not being driven externally. It is present for use in some other part of the accelerating cycle or, in multi-purpose accelerators, to accelerate some other species of particles. If the cavity has an eigenfrequency near a harmonic of the beam circulation frequency, a resonance condition exists that can lcad to azimuthal bunching of the beam. It must be emphasized that any eigenmode of the cavity, not just the fundamental mode, is a potential source of this effect.

The effect is closely related to the negative mass effect discussed above. We can define a quantity q analogous to the quantity $\mathrm{g}$ in $\mathrm{Eq}$. (1). This quantity is directly proportional to the shunt impedance $Z$ of the particular mode of the cavity near resonance. If the cavity mode is inductively cletuned from a harmonic of the circulation frequency, $q$ is negative and a beam below transition energy may be unstable, while a beam above transition is stable. If the cavity mode is capacitively detuned, $q$ is positive and the situation is reversed. The lower limit on the frequency spread in the beam contains the product of the shunt impedance and the beam current. The most stringent criterion, which is sufficient for stability either above or below transition, is given by

$$
\frac{Z}{Z_{s}}<\frac{2 \pi n c(\Delta E)^{2} l_{0} \omega_{c / \partial E !}}{\left(m_{0} c^{2}\right) r_{0} N \omega_{c}^{2}}
$$

in which $Z_{S}$ is the impedance of free space (equal to $377 \mathrm{ohms}$ ), $m_{0}$ is the rest mass of the particles, and $r_{0}$ is the classical radius of the particle. In writing this condition, it has been assumed that the frequency spread arises from energy spread alone and $\Delta \mathrm{E}$ is the energy spread necessary for stability. Additional frequency spread can result from the spread in betatron amplitudes.

The effect described above can also occur for bunched beams. A beam being accelerated by the fundamental mode of an rf system may encounter resonances with a higher mode of that system, or some mode of another system if one is present. Care should be taken to avoid such resonances and if this is not possible, to kecp the shunt impedances at sufficiently low values.

\section{Transverse Resistive Wall Instability}

An instability attributed to the finite resistance in the walls of the vacuum tank was first investigated by the MURA group in the $50-\mathrm{MeV}$ electron accclerator. The instability manifests itself by vertical oscillations of the azimuthally uniform beam with frequencies $\omega=(n-\nu) \omega_{c}$, where $n$ is an integer, $v$ is the verticle betatron wave number, and $\omega_{c}$ is the circulation frequency. The disastrous consequences of this phenomena are obvious - the beam hits the wall. This observation prompted a theoretical investigation that first treated the effect in an azimuthally uniform beam. The treatment included the stabilizing effects of spread of the appropriate frequency, but the calculation was not self-consistent. Later, the work was extended to a treatment of bunched beams, and this later work included one of the spreads expected to contribute to stabilization.

\section{Azimuthally Uniform Beams}

The physical explanation of the destabilizing effect of finite resistance in the walls may be obtained on the basis of a simple model. Consider a beam of particles with directed velocity in the $z$ direction located between two conducting surfaces, as shown in Fig. 2. Suppose the beam is

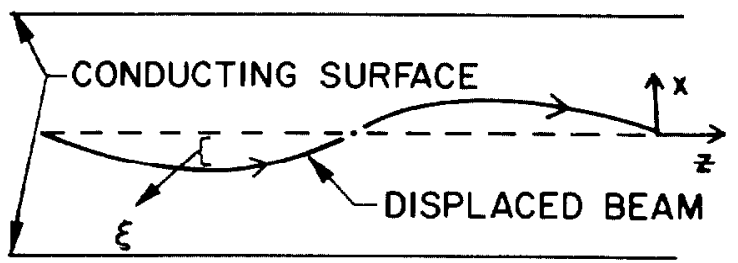

Fig. 2 Position of displaced beam at a given instant of time.

subject to a small perturbation that shifts its position from $x=0$ to $x=\Delta$, with $\Delta$ of the form

$$
\Delta=\xi \mathrm{e}^{\mathrm{i}(\mathrm{kz}-\omega \mathrm{t})}
$$

where $\xi$ is a constant. If the conductivity of the surfaces is infinite, the force $F$ on the beam in the $x$ direction is just proportional to the displacement so that $F-\int \Delta$, $\Gamma$ beirg a function of the beam current and the distance to the walls. Image charges in the walls tend to pull the beam toward the nearest wall, and the image currents tend to push it away. The charges win out so that the force is in the direction of the displacement. The velocity of the beam in the $x$ direction is $v_{b}=-i \omega \Delta$, while the particles in the beam have a velocity component in the $x$ direction $v_{p}=i\left(k v_{z}-\omega\right) \Delta$. Thus if $v_{Z}>\omega / \mathrm{k}(i . e .$, if the particles - directed velocity is greater than the phase velocity of the wave), the beam and the particles have oppositely directed $x$ velocity. If the wall conductivity is infinite the force is $90^{\circ}$ out of phase with the particle velocity, and no work is done on the particles. The wave is therefore stable. If the wall conductivity is finite, the image charges tend to lag behind, and the force takes the form $F=(f+i h) \Delta$. Now if $v_{7}>\omega / k$, the force has a component in phase with the particles velocity. Energy goes into the transverse motion of the particles and the wave is unstable. The energy comes ultimately from the directed motion of the particles. This effect is a transverse analogue of that utilized in the resistive wall amplifier.

The theory for unbunched beams contains two frequencies, $U$ and $V$, where $U$ is proportional to the component of the transverse force that is in phase with the beam displacement, and $V$ is proportional to the component that is $90^{\circ}$ out of phase. Expressions for $U$ and $V$ depend upon the geometry 
of the beam and the vacuum tank, but the simplest form will suffice for an illustration. Consider a beam with uniform density and circular cross section of minor radius a inside a pipe with minor radius $b$. The beam is rigidly displaced by an amount $\Delta$, with $\Delta$ of the form given by $\mathrm{Eq}$. (5). The expressions for $U$ and $V$ are

$$
\begin{gathered}
U=-\frac{e^{2} \mathrm{~N}\left[1-(\mathrm{a} / \mathrm{b})^{2}\right]}{2 \pi \nu_{0} \omega_{c 0} \gamma^{3} \mathrm{~m}_{0} \mathrm{Ra}^{2}}, \\
V=\left(e^{2} \mathrm{~N} \beta / \pi \nu_{0} \gamma \mathrm{m}_{0} \mathrm{~b}^{3}\right)(8 \pi \sigma \operatorname{Re} \omega)^{-1 / 2},
\end{gathered}
$$

where $\nu_{0}$ and $\omega_{\mathrm{C} 0}$ are the ave rage values of $\nu$ and $\omega_{c}, \sigma$ is the conductivity of the wall material, and $\beta=\omega_{c 0} R / c$. The value of the real part of $\omega$ is to be taken from $\mathrm{Fq}$. (9).

In the absence of any spread in $\nu$ or $\omega_{c}$, the growth rate (e-folding time) is just $V$. Except for extremely relativistic beams, the condition $U \gg V$ holds. The criterion for stabilization by Landau damping places a lower limit on the spread in the quantity $\mathrm{S}=(\mathrm{n}-\nu) \omega_{\mathrm{c}}$. Both $v$ and $\omega_{c}$ are, in general functions of particle energy $\mathrm{E}$ and betatron oscillation amplitude $\rho$. The lower limit on the spread is $\Delta S=K|U|$, where $K$ is again a constant of order unity whose exact value depends on the distribution of particles in $F$, and $\rho$. If the condition $U \gg>$ does not hold, the criterion can be written as

$$
\Delta S=K \sqrt{U^{2}+V^{2}}
$$

The theory predicts that only perturbations with $n>v$ are unstable in a uniform beam, and the real part of the frequency is given by

$$
\operatorname{Re} \omega \approx\left(n-v_{0}\right) \omega_{\mathrm{c} 0}
$$

These predictions are in agreement with experiment, but the growth rates and $\Delta S$ values required for stability are orders of magnitude different from those observed in the MURA accelerator. This discrepancy has apparently been explained by treating the effect of the clearing electrodes. The presence of these circuits, not the resistance of the aluminum walls, may be the dominant effect in that machine. Changing the termination of the clearing electrode circuits has a drastic effect on the experiment.

Equation (8) suggests that is is impossible to cure this instability by use of passive circuits alone because no passive circuit can cancel the effect of the resistance (i.e., reduce $V$ to zero). However, walls can be devised that greatly reduce the value of $L$ and even change its sign (see Ref. 6). These walls might then greatly reduce the spread necessary for stabilization. It is not always convenient to introduce additional energy spread in a beam, but it is possible, through the use of octupole magnets, to introduce significant nonlinearity in the betatron motion. This nonlinearity contributes to the spread in $\mathrm{S}$ by enhancing the dependence of $\nu$ on the betatron amplitude.
This method of stabilization has been used in several machines, and in at least one instance the degree of success was extremely gratifying.

As mentioned earlier, a useful method of stabilizing transverse oscillations is by means of electronic feedback. This method has been successful in suppressing the instability in both azimuthally uniform (unbunched) and bunched beams. However, this method should not be relied upon too heavily. The effectiveness of the method depends to some extent on the slow growth rate as well as the rather low frequencies characteristic of the motion. Shorter wavelength perturbations may not be so easily damped, as the frequencies become so high as to tax the electronic feedback system.

\section{Bunched Beams}

The instability as observed in bunched beams violates one of the principal results of the theory described above, namely, it was found that modes with $\mathrm{n}<\nu$ can also be unstable. Another experimental observation was that a spread in particle energy did not seem to be effective in suppressing the instability. It has been postulated, but not confirmed theoretically, that energy spread is not effective because this contribution to the spread in $\mathrm{S}$ averages out if the bunch moves as a single body. On the other hand, it seems reasonable that energy spread would be very effective for stabilizing internal motion of the bunch. No theoretical analysis of internal transverse motion has so far appeared, although strange effects have been observed experimentally.

Although the resistance in the walls is the same ultimate cause of the instability in bunched beams, it manifests itself in a slightly different way. Consider a particle moving inside a straight pipe and oscillating transversely so that its displacement relative to the axis of the pipe is of the form $\xi \exp \left(i v \omega_{c} t\right)$. The frequency is below the cutoff frequency of the pipe. Because of the finite resistance in the walls of the pipe, one component of the electromagnetic field of this particle. namely the magnetic ficld orthogonal to the axis and the displacement, falls off as $d^{-1} / 2$, where $d$ is the distance behind the particle. ${ }^{8}$ This residual field at any point does not oscillate in time, but has the same phase as that of the displacement of the particle when it passed that point. In the straight pipe the particle is subject only to the fields where it is, and it can be shown that thesc fields are such as to damp the oscillations.

Now let the pipe be bent in a big circle to form an accelerator vaccum tank. The oscillating particle will feel the damping influence of the local fields, but each time around it also feels the effect of the residual fields it left there on previous turns. The effect of the residual fields is larger than that of the local field. It is easy to see that thesc residual fields will be stabilizing or destabilizing, depending upon the change in phase of the displacement during one turn around the machine. Thus, the condition for stability is a condition on the valuc of $v$, and this turns out to be $I<\nu<I+\frac{1}{2}$, where $I$ is any integer. A 
simple derivation of this result is given in Ref. 1 and a more comprehensive treatment is presented in Refs. 3 and 4. Unfortunately, no clear-cut experiment has been performed to verify this result. Such an experiment would require an accelerator that contains one bunch, exhibits the instability, and is capable of a variation in $v$ across a half integer.

It is interesting to compare the growth rate of the instability for a single bunch of $N$ particles with that for a uniform beam of the same number of particles. If the growth rate for a single bunch is calculated neglecting the local fields (a good approximation), then the growth rate, $\operatorname{Im} \omega$, is given by

$$
\operatorname{Im} \omega=V_{n} \sqrt{2(n-\nu)}|\operatorname{Im} G|
$$

where $V_{n}$ is the growth rate for the perturbation with $\mathrm{n}$ wavelengths in the uniform beam as found rrom Eq. (7). We have used also Eq. (9) in writing Eq. (10). The quantity $G$ is a function of $\nu$ only, with Im G| increasing from zero at $v=I-1 / 2$ to unity at $v=I-1 / 4$. The point is, on the basis of this simple theory, if the beam is bunched, the growth rate has the same dependence on parameters (ex cept $v$ ) as that for an unbunched beam. In practice, even the numerical difference is not very great.

The work of Ref. 4 contains an analysis of the instability in beams with many bunches. If the bunches are equally spaced and have equal number of particles, then a simple selection rule holds that determines which modes are stable and which are unstable. If there are $\mathrm{M}$ bunches, they can oscillate coherently in only $M$ normal modes. If $n$ is the number of wavelengths around the machine $(0 \leq n<M)$, then the modes such that $I<(\nu+1) / \mathrm{M}<\mathrm{I}, \frac{1}{2}$ are stable, again with $\mathrm{I}$ any integer. Of course, no accelerator has such equal bunches so the question arises, how different does the number of particles per bunch have to be in order that the bunches cease to act coherently and go their independent way? A criterion is presented in Ref. 4, but it depends on quantities analogous to the $U$ and $V$ discussed above. The quantity $\mathrm{U}$ is very hard to calculate accurately, even for an unbunched beam. It depends on the exact form of the local fields, which in turn are a function of the shape of the bunch as well as the configuration of the vacuum tank. Taken as a whole, the experimental results seem to favor independent motion. In particular, observations in the NIMROD machine indicate that independent motion sets in when a $10 \%$ variation exists in bunch population. Attempts to calculate the spread in $v$ necessary for stability suffer the same pitfall. The spread necessary depends strongly on that elusive quantity $\mathbf{U}$.

\section{Storage Rings}

The term storage ring applies to two types of devices, the first of which is simply a machine to store a single beam until it is wanted. This type of device may be more subject to instabilities than an accelerator because of the perhaps longer time the particles are in the machine. But the effects are the same in that the cause of the instabilities is the interaction of the beam with itself and its surroundings through its coherent self-fields.

The second category of storage rings are those devices that contain two beams, either in une or two rings. Whether there are one or two rings, the beams meet head-on one or more times each turn. In addition to the interaction of a beam with its own fields, it also interacts with the coherent fields of the other beam. The selfelectric and self-magnetic fields of a single beam usually tend to cancel each other, resulting in a factor $\gamma^{-2}$ in most of the forces exerted on the particles [e.g., Eqs. (1) and (6)]. But the effects of the electric and magnetic fields of the other beam do not cancel - they add. Thus the beambeam forces are a factor $2 \gamma^{2}$ bigger than the single-beam forces. For high-energy particles, especially electrons (because $\gamma$ is so large), the beam-beam interaction completely dominates in determining both the coherent and incoherent stability of the particles. As the two beams collide, all particles suffer a sudden change in their transverse velocity, resulting in a change in betatron amplitude. This sudden change can cause incoherent loss of all, or a portion of, either or both beams. The so-called "low $\beta$ " concept postulated to alleviate this catastrophic loss is discussed in paper E-20 of this conference.

Effects of the beam-beam interaction on the coherent motion of the two beams is the subject of an investigation by Sessler and Pellegrini. This work is not yet complete, but it reveals certain selection rules such as those presented above for one beam. Experimentally, it has been found that making the $\nu$ values of the two beams sufficiently differcnt suppressed unstable vertical oscillations in the Stanford electron storage rings.

A study of instabilities that result in a distortion of the beam cross section has been undertaken by Lee, Mills, and Morton, who present some results in paper E-14. These distortion modes could arise from the interaction of two beams. rhey would be harder to detect than the coherent transverse motion, and generally not susceptable to stabilization by feedback.

\section{Conclusions}

The theory of coherent beam instabilities is not complete, rigorous, or even self-consistent in most cases. The theory should be extended and widened in scope, and certainly more experimental results are needed. Experimental data regarding these effects is sometimes hard to obtain. For one reason, physicists seldom look for an instability in their machines; they wait until one occurs, then investigate it. But gathering data is not easy since the machine may not be equipped with the necessary diagnostic tools. Accelerators are built to accelerate particles, not to study instabilities. There are exceptions in the MURA electron accelerator and the new electron storage ring at that facility. The latter 
is being built with the express purpose of investigating beam behavior.

But I believe that the theory is basically sound. It can be used, and has been used, as a predictive tool. Methods suggested by the theory have been successful in suppressing instabilities as they occur in numerous machines. The theory also offers guidelines for the design of new machines. As an example, if a new high-intensity machine with a single bunch were to be built, it would be highly desirable to satisfy the condition $I<\nu<I+\frac{1}{2}$.

Further, theory can display warning signs regarding new concepts and new components. In this respect, and leading to the subject of future work, consider the ceramic vacuum tank.

Ceramic material has two very attractive features: it has excellent vacuum properties, and the conductivity is so low that even in rapid-cycling machines the magnetic guide field easily penetrates the vacuum vessel. But to my knowledge little thought has been given to the effects of such a vacuum tank on the coherent motion of the beam. Certainly the existing theory should be extended to accommodate these effects. The chief worry here is that the ceramic walls are relatively transparent to the fields arising from coherent motion. Thus the fields reach out into empty space or terminate on the lossy material of the magnets, with perhaps unwanted consequences. There does exist the possibility of coating the ceramic on the outside with a thin layer of highly conducting material. This layer would hopefully let the guide field penetrate, but still contain the relatively high-frequency fields from any coherent motion. The feasibility of this scheme can be determined for any individual machine.

Another topic that should be investigated is the effect of the background gas on beam behavior. Many of the experimental results show a pressure dependence of growth rates and thresholds, so clearly the "surroundings" of the beam must include the residual gas through which it passes. This effect is not an easy one to investigate - it is, in effect, a beam-plasma problem.
'The relatively untouched question of motion within a bunch is one not easily handled analytically. Some calculations have been performed with computers, and with the ever-increasing capability of computers and computational techniqucs, a great deal more could be done. In particular, the postulates regarding the stabilizing effect of energy spread within the beam should be investigated.

Devising new walls, circuits, and general gadgetry for suppressing known instabilities (as well as those yet to be discovered) is a challenge to the inventive genius of physicists and engineers alike. This very important, and relatively new, approach to stability problems will no doubt reccive much attention in the future.

\section{References}

1. A. M. Sessler, Proc. V Intern. Conf. High-Energy Accelerators, Frascati, 1965 (CNEN, Rome, 1966) p. 319.

2. R. W. Landau and V.K. Neil, "Negative Mass Instability," CCRL-14406 Rev 1, 1966 . To be published in Phys. Fluids.

3. N. S. Dekanskij and A. N. Skrinski, "Coherent Instability of Bunches of Charged" Particles," Inst. Nucl. Phys. Rept. Novosibirsk, IISSR, 1965 (unpublished).

4. E. D. Courant and A. M. Sessler, Rev. Sci. Instr. 37, 1579 (1966).

5. L. J. Laslett, Proc. Intern. Symp. Electron and Positron Storage Rings, Saclay, 1966.

6. R. J. Briggs and V.K. Neil, J. Nucl. Energy Part C, $\underline{8}, 255$ (1966).

7. V.K. Neil and R. J. Briggs, "Stabilization of Nonrelativistic Beams by Means of Inductive Walls" UCRL-70133, Sept. 14, 1966. To be published in J. Nucl. Energy, Part C.

8. P.L. Morton, V.K. Neil, and A.M. Sessler, J. Appl. Phys. 37, 3875 (1966). 